\title{
Nation-wide prospective surveillance of Clostridium DIFFICILE INFECTIONS IN HOSPITALS IN BELGIUM, JULY 2007 -JUNE 2008
}

\author{
M L Lambert (mllambert@iph.fgov.be) ${ }^{1}, K_{\text {Mertens }}^{1}$, I Ramboer ${ }^{1}$, M Delmée $^{2}$, C Suetens ${ }^{1,3}$ \\ 1. National Surveillance of Infections in Hospitals (NSIH), Epidemiology Unit, Institute of Public Health, Brussels, Belgium \\ 2. National reference laboratory for Clostridium difficile, St Luc Hospital, Catholic University Leuven, Brussels, Belgium \\ 3. Current address: European Centre for Disease Prevention and Control (ECDC), Stockholm, Sweden
}

\begin{abstract}
We report here baseline data from the first year of compulsory surveillance of Clostridium difficile infections (CDI) in hospitals in Belgium. Between 1 July 2007 and 30 June 2008, 2,704 CDI were reported: $12 \%$ were recurrent and $66 \%$ were hospital-associated (half of which occurred 15 days or more after admission). CDI was considered the cause of death (direct or indirect) for $10 \%$ of the episodes. The median incidence of CDI was 1.5 per 1,000 admissions and 1.9 per 10,000 hospital-days for all cases, and 0.9 per 1,000 admissions, and 1.1 per 10,000 hospital-days for hospital-associated cases. Further investigation of risk stratification by average length of stay in the reporting hospitals is warranted as a way to improve the comparability of indicators across hospitals and surveillance systems. In spite of methodological issues, the surveillance of CDI in Belgian hospitals has been able to produce robust baseline data that should allow monitoring of trends at hospital and national level, and provide a basis for international comparisons. Remaining challenges are to define and monitor targets for the control of CDI, and to improve the individual feedback of data at hospital level.
\end{abstract}

\section{Introduction}

Clostridium difficile associated infection (CDI) is now recognised as a major cause of morbidity and mortality in hospitals [1]. Robust data are key for setting accurate targets to control the disease and for monitoring how these targets are achieved, but to our knowledge, no incidence data originating from prospective nationwide surveillance systems have yet been published in peer-reviewed journals.

In this article, we report baseline data from the first year of compulsory surveillance of $\mathrm{CDI}$ in hospitals in Belgium in the period from July 2007 to June 2008. These data include a basic description of $\mathrm{CDI}$ and the distribution of incidence rates across reporting hospitals. The essence of a surveillance system is to provide comparable data, i.e. data that can be compared over time, between hospitals and between surveillance systems. We also discuss methodological issues such as the importance of declaring zero cases and the risk stratification of hospitals per length of stay.

\section{Materials and methods}

Prospective surveillance of CDI in hospitals in Belgium started in July 2006 and has since 1 July 2007 been compulsory by federal law for all hospitals, with the exception of psychiatric hospitals and hospitals providing chronic care of less than 150 beds (although their voluntary participation is encouraged). Hospitals are requested to report all their CDI over at least one six-month period per year (termed in this article first and/or second "semester"). Therefore the unit of our analyses is a "hospital semester", although some hospitals contributed data for the entire year. Registration and data entry are web-based and are separate processes. There is no explicit requirement to declare zero cases, making it impossible to differentiate between "no cases" and "no reporting".

Case-definitions for CDI, hospital-associated (HA) case and recurrent case follow recommendations from the European Working Group on $C$. difficile [2]. Hospital data are limited to denominators from which the average length of stay can be calculated (number of admissions per number of hospital-days). Incidences are computed as number of CDI per 1,000 admissions (or cumulative incidence), and as number of CDI per 10,000 hospital-days (or incidence density).

Stata 10 statistical package was used for data analysis.

Results

From 1 July 2007 to 30 June 2008, 130 hospitals registered $2,704 \mathrm{CDI}$ over 229 surveillance semesters. We excluded 31 of the 229 (14\%) surveillance semesters, for which no denominator data were available. This left 198 surveillance semesters (120 hospitals) for the analysis of incidence rates.

\section{Description of cases}

Of $2,704 \mathrm{CDI} 58 \%$ occurred in female patients, $12 \%$ were recurrent cases and $66 \%$ occurred more than two days after admission (hospital-associated CDI). CDI was considered as cause of death (direct or indirect) for $10 \%$ of the cases. Median age was 78 years, $75 \%$ of cases were in patients 65 years-old or older.

Most CDI occur late in the course of the hospital stay: $75 \%$ of HA cases occurred eight days or more after admission (Figure 1).

\section{Description of hospital-semesters}

Table 1 shows the distribution of 198 hospitals semesters in terms of size of the hospitals (number of beds, admissions), length of stay and total hospital-days during the surveillance period. There are clear outliers in the distribution. 
For 37 of the198 hospital-semesters (19\%), no cases were reported. CDI being a rare event, it is plausible for small hospitals to have zero cases over six months, but less so for larger hospitals. However 20 (54\%) of the 37 hospital-semesters without cases were in hospitals reporting more than 30,000 hospital-days for the semester.

\section{Incidences}

The distributions across hospital-semesters are shown in Table 2 and Figure 2 (note that many hospitals contributed data for the full year).

The median incidence of HA-CDI across hospitals in Belgium was 1.1 cases per 10,000 hospital-days, but the figure shows clearly that the mode was below one case per 10,000 hospital days, which is partly due to the large number of hospitals not reporting cases (whether zero case, or not reporting). It further shows outliers, which presumably reflect outbreaks, including a very severe one (12 cases per 10,000 hospital-days).

We compared characteristics of hospital-semesters and incidences of HA CDI across hospital-semesters, categorised according to their average length of stay.

\section{F I G U R E}

Time from hospital admission to beginning of CDI in hospitalassociated cases $(n=1,794$ cases occurring more than two days after admission)

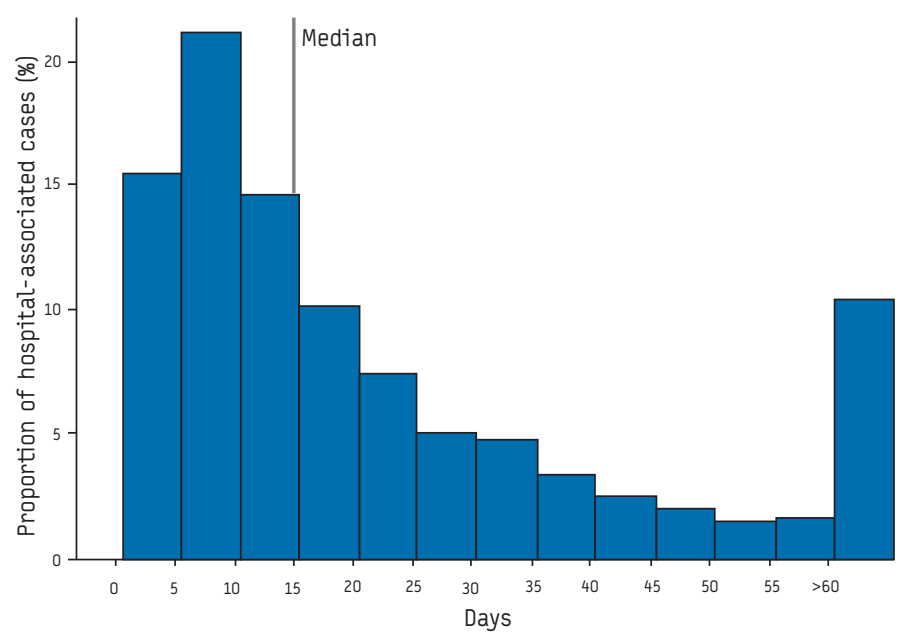

CDI: Clostridium difficile infections

\section{T A B L E 1}

Characteristics of hospitals contributing data for CDI surveillance in Belgium, July 2007-June $2008(n=198$ hospital-semesters)

\begin{tabular}{|l|c|c|c|c|c|c|}
\hline Percentiles & P10 & P25 & P50 & P75 & P90 & Max \\
\hline Beds & 126 & 197 & 294 & 467 & 822 & 1,809 \\
\hline Average length of stay [days] & 6.2 & 6.8 & 7.8 & 9.6 & 13.7 & 127.5 \\
\hline Admissions & 1,170 & 3,168 & 5,040 & 7,758 & 13,871 & 32,145 \\
\hline Hospital-days & 17,488 & 27,389 & 37,706 & 59,962 & 106,774 & 248,112 \\
\hline
\end{tabular}

CDI: Clostridium difficile infections
This table shows that hospitals with patients staying for an average period of 14 days or more tend to be smaller than those with a length of stay shorter than 14 days. They more often do not report CDI cases, but when they do, report higher rates.

\section{Discussion}

The median incidence of all CDI across Belgian hospitals in the period from July 2007 to June 2008 was 1.5 per 1,000 admissions, an incidence higher than the median of 1.1 per 1,000 admissions reported in the first European survey (212 laboratories in eight countries surveyed in the year 2000) [3]. By contrast, the median incidence of all CDI in 35 hospitals in Germany in 2007 was 5.6 per 10,000 hospital-days [4], compared to 1.9 in Belgium (July 2007-June 2008). In Germany, the proportion of hospital-associated cases was $73 \%$, compared to $66 \%$ in Belgium. In England, the mean incidence of all CDI in patients 65 years-old or older ranged from 17.5 to 27.4 per 10,000 hospital-days in 2007, depending on the region [5]. In a representative sample of community hospitals in the United States, the mean CDI incidence was $1.3 \mathrm{CDI}$ per 1,000 discharges in 2005 (ICD_9 code 0845) [6]. Overall, the data available for comparisons (i.e. data from a representative sample of hospitals) are rather scarce.

It is noteworthy that $50 \%$ of $\mathrm{HA}$ cases occurred more than 15 days after admission, reflecting not only the risk associated with a longer exposure [7], but also presumably the fact that a longer period of stay is a proxy for the severity of the condition of the hospitalised patients, in itself a risk factor for CDI.

Since there is no requirement to explicitly report zero cases, hospitals that do not report at all are included in the evaluation as reporting zero cases. CDI being a rare event (in the absence of outbreaks), having zero cases over a period of six months is plausible in small hospitals but less likely in larger hospitals, and our data are therefore an underestimation of the real rates, because for these hospitals reporting no case more likely indicates no reporting. This shows the importance of explicit reporting of zero cases, which we are now implementing in Belgium (hospitals registering online for a new surveillance period, will be requested to actively declare closure of the previous reporting period, with or

T A B L E 2

Incidences of CDI in hospitals in Belgium, July 2007-June 2008 (for 198 hospital-semesters)

\begin{tabular}{|c|c|c|c|c|}
\hline & \multicolumn{2}{|c|}{ All cases } & \multicolumn{2}{|c|}{ Hospital-associated cases } \\
\hline & $\begin{array}{l}\text { per } 1,000 \\
\text { admissions }\end{array}$ & $\begin{array}{c}\text { per } 10,000 \\
\text { hospital-days }\end{array}$ & $\begin{array}{l}\text { per } 1,000 \\
\text { admissions }\end{array}$ & $\begin{array}{c}\text { per } 10,000 \\
\text { hospital-days }\end{array}$ \\
\hline \multicolumn{5}{|c|}{ Mean of means } \\
\hline & 2.5 & 2.3 & 1.9 & 1.5 \\
\hline $95 \%$ CI & $1.8-3.3$ & $2.0-2.5$ & $1.2-2.5$ & $1.3-1.7$ \\
\hline \multicolumn{5}{|c|}{ Percentiles } \\
\hline $\mathrm{P} 10$ & 0.0 & 0.0 & 0.0 & 0.0 \\
\hline P25 & 0.6 & 0.7 & 0.3 & 0.4 \\
\hline P50 & 1.5 & 1.9 & 0.9 & 1.1 \\
\hline P75 & 2.8 & 3.4 & 1.9 & 2.3 \\
\hline P90 & 4.8 & 4.5 & 3.1 & 3.3 \\
\hline \multicolumn{5}{|c|}{ Maximum } \\
\hline & 56.4 & 13.9 & 46.7 & 12.0 \\
\hline
\end{tabular}

CDI: Clostridium difficile infections; CI: confidence interval. 
without cases). On the other hand, given that surveillance of CDI in Belgium is compulsory for only one half of a year, we cannot exclude that hospitals would prefer to report on semesters with higher CDI incidence, or outbreak. This would lead to an overestimation of the real rates at national level. In Belgium, hospitals with a longer average period of stay tend to be smaller than those with a shorter stay: our data show that they are more likely not to report cases, but they are also more likely to report high rates when reporting cases, supporting the hypothesis of a bias towards reporting in case of outbreaks. Finally, 13\% of surveillance semesters were excluded from the calculation of rates because of missing denominator data, but we do not know if this created a bias, or not.

An issue particularly important for international comparisons is the different mix of hospitals contributing to the computation of national CDI incidence estimates. In Belgium, "acute" hospitals accommodate a varying number of beds for chronic patients. In that respect, stratifying hospitals based on patients' average length of stay might be more appropriate than a stratification of acute versus

\section{F I G U R E 2}

Incidences of hospital-associated CDI in hospitals in Belgium, July 2007-June 2008 ( $n=198$ hospital-semesters)

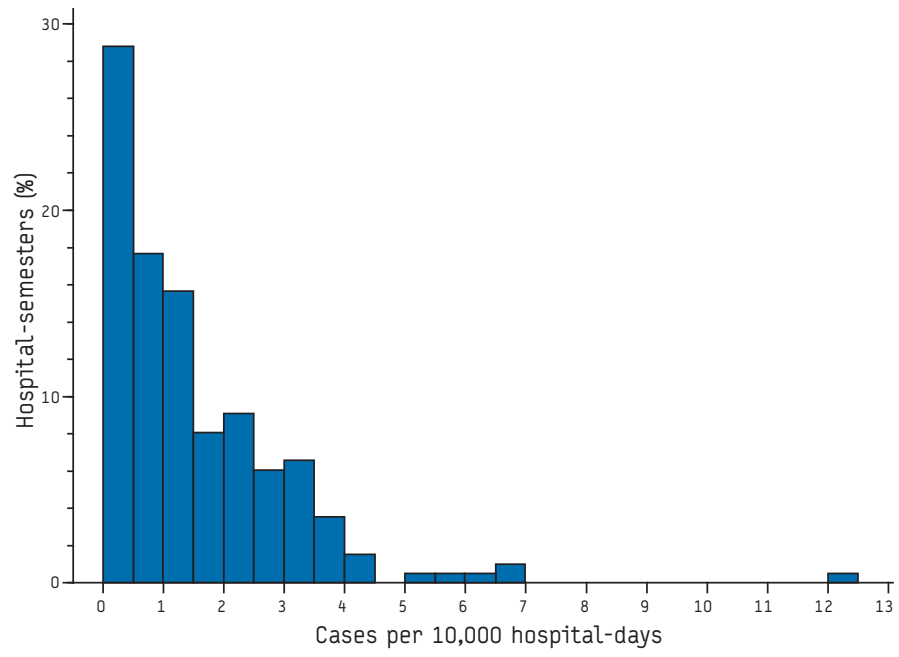

CDI: Clostridium difficile infections chronic hospitals based on administrative definitions at national level. We intend to investigate further whether risk stratification by average length of stay of the reporting hospital is a way to improve the comparability of indicators across hospitals, and across surveillance systems.

\section{Conclusions}

Although it raises methodological issues, such as the need for an explicit reporting of zero cases, the surveillance of CDI in hospitals in Belgium has been able to produce robust baseline data that can improve our understanding of the epidemiology of the disease. It should also allow for a monitoring of trends at hospital and national level and provide a basis for international comparisons. Remaining challenges are to define and monitor targets for the control of CDI, and to improve the usefulness of data at hospital level through result-oriented individual feed-back.

\section{References}

1. Suetens C. Clostridium difficile: summary of actions in the European Union. Euro Surveill. 2008;13(31):pii=18944. Available online: http://www. eurosurveillance.org/ViewArticle.aspx?ArticleId=18944

2. Kuijper EJ, Coignard B, Tüll P; ESCMID Study Group for Clostridium difficile; EU Member States; European Centre for Disease Prevention and Control. Emergence of Clostridium difficile-associated disease in North America and Europe. Clin Microbiol Infect. 2006;12 Suppl 6:2-18.

3. Barbut F, Delmée M, Brazier JS, Petit JC, Poxton IR, Rupnik M, et al. A European survey of diagnostic methods and testing protocols for Clostridium difficile. Clin Microbiol Infect. 2003;9(10):989-96.

4. KISS Krankenhaus-Infektions-Surveillance-System. Modul CDAD-KISS. [KISS Hospital Infections Surveillance System. Module CDAD-KISS ]. Berlin: National Reference Centre for Surveillance of Nosocomial Infections; 2008 Sept 9. Availabe from: http://www.nrz-hygiene.de/dwnld/CDAD_Referenzdaten_2007. pdf. [In German].

5. Health Protection Agency. Surveillance of Healthcare Associated Infections Report: 2008. London: Health Protection Agency; July 2008. Available from: http://www.hpa.org.uk/web/HPAwebFile/HPAweb_C/1216193833496

6. Zilberberg MD, Shorr AF, Kollef MH. Increase in adult Clostridium difficilerelated hospitalizations and case-fatality rate, United States, 2000-2005. Emerg Infect Dis. 2008;14(6):929-31.

7. Barbut F, Petit JC. Epidemiology of Clostridium difficile-associated infections. Clin Microbiol Infect. 2001;7(8):405-10.

This article was published on 9 April 2009.

Citation style for this article: Lambert ML, Mertens K, Ramboer I, Delmée M, Suetens C. Nation-wide prospective surveillance of Clostridium difficile infections in hospitals in Belgium, July 2007-June 2008. Euro Surveill. 2009:14(14):pii=19169. Available online: http://www.eurosurveillance.org/ViewArticle.aspx?ArticleId=19169

T A B L E 3

Hospital characteristics and incidence of CDI , by average length of stay of reporting hospitals. Belgium, July 2007-June 2008

\begin{tabular}{|c|c|c|c|}
\hline Average length of stay & $<14$ days & ${ }^{3} 14$ days & \\
\hline Hospital-semesters (n) & 181 & 17 & \\
\hline Hospital-semesters not reporting cases (n) & $31(17 \%)$ & $6(35 \%)$ & $\mathrm{Chi}^{2}=3.4, \mathrm{p}=0.07$ \\
\hline Number of beds, median & 319 & 166 & \\
\hline Number of admissions, median & 5,239 & 503 & \\
\hline Number of hospital-days per semester, median & 39,357 & 20,350 & \\
\hline \multicolumn{4}{|l|}{ HA CDI per 10,000 hospital-days } \\
\hline P25 & 0.4 & 0.0 & \\
\hline P50 & 1.1 & 1.1 & \\
\hline P75 & 2.2 & 3.5 & \\
\hline Hospital-semesters with more than three HA cases per 10,000 hospital-days & $23(13 \%)$ & $6(35 \%)$ & $\mathrm{Chi}^{2}=6.3, \mathrm{p}=0.01$ \\
\hline
\end{tabular}

